\title{
Wie Friedrich List (1789-1846) den Brexit und den Verhandlungsmarathon zwischen Großbritannien und der EU kommentieren würde
}

\author{
Eugen Wendler
}

Angenommen: 21. Januar 2021 / Online publiziert: 10. Februar 2021

(C) Der/die Autor(en) 2021

Zusammenfassung In buchstäblich letzter Minute haben sich die englische Regierung und die Europäische Union auf ein umfangreiches Abkommen geeinigt, um einen ungeregelten Brexit zu verhindern. Nach dem jahrelangen zähen Verhandlungsmarathon fällt der Jubel verhalten aus, dennoch herrscht auf beiden Seiten des Ärmelkanals Erleichterung, weil ein Modus Vivendi gefunden wurde, auf dem sich die künftigen Beziehungen aufbauen und fortführen lassen. Ob sich die englischen Blütenträume, die an den Brexit geknüpft wurden, erfüllen werden, wird die Zukunft erweisen.

Die Strategie und Taktik der englischen Regierungen zum Brexit und bei den Austrittsverhandlungen spiegeln sich in den Erfahrungen wider, die Friedrich List vor genau 175 Jahren bei seinen Bemühungen um eine deutsch-englische Allianz machen musste. Wegen der von England schon damals strikt befolgten Insular- und Handelssuprematie musste er sich eingestehen, dass England diese Position hartnäckig verteidigt und deshalb frustriert und ernüchtert seine Pläne aufgeben. Deshalb setzte er seine Hoffnung auf eine „Kontinentalallianz“ der europäischen Nationen, wie sie nun nach dem Austritt Großbritanniens aus der Europäischen Union entstanden ist. Vielleicht werden wir uns nun an den Begriff „Kontinentalallianz“ gewöhnen müssen und dabei an die Weitsicht von Friedrich List erinnert.

Andererseits gilt auch für die englische Politik das Motto von Lists zweiter Pariser Preisschrift: „Le monde marche - Die Welt bewegt sich“, allerdings mit völlig anderen Vorzeichen als vor 175 Jahren: Die Welthandelsachse hat sich von der westlichen auf die östliche Halbkugel verlagert; das britische Weltreich ist Geschichte, die Fließgeschwindigkeit des globalen Wandels hat sich dramatisch beschleunigt und trotz der Lingua Franca erscheint England, vor allem aus asiatischer Sicht, nur noch als kleiner Fleck auf der Weltkarte. Falls die schottische Regierung ihre Ab-

E. Wendler $(\bowtie)$

Hochschule Reutlingen, Reutlingen, Deutschland

E-Mail: eugen-wendler@web.de 
sicht durchsetzen und die Unabhängigkeit vom Vereinigten Königreich erreichen sollte, würde sich der Brexit als verhängnisvoller Bumerang erweisen.

Schlüsselwörter Brexit · Kontinentalallianz · Friedrich List und seine Vision einer deutsch-englischen Allianz · Die englische Insular- und Handelssuprematie · Die britsche Handelsstrategie und Taktik

\title{
How Friedrich List (1789-1846) would comment on Brexit and the negotiation marathon between Great Britain and the EU
}

\begin{abstract}
At the last minute, the British Government and the EU agreed on a comprehensive trade agreement to avert an unregulated Brexit. After the years of negotiations, the cheers are muted; nevertheless, there is relief on both sides of the Channel, because a modus vivendi has been found on which future economic, political and cultural relations between Great Britain and the EU can be built and continued. Then it will show whether the blossoming dreams that England is hoping from Brexit will come true.

The strategy and tactics of the British Government during Brexit and the exit negotiations are reflected in the experience Friedrich List had to make exactly 175 years ago in his effort to establish a German-English alliance. Because of England's strict insular and trade supremacy he had the bitter experience of England stubbornly defending this position. Therefore, in his visions, he hoped for a "continental alliance" of European nations, as it emerged after Great Britain left the European Union. Perhaps we will now have to get used to the term "continental alliance" and are reminded of the visionary wisdom of Friedrich List.

On the other hand, the motto of List's second Paris prize publication "Le monde marche - the world is moving" also applies to England politics; but with completely different signs than 175 years ago. The world trade axis has shifted from the western to the eastern hemisphere, the Empire is history, the fluidity of global change has accelerated dramatically and despite the lingua franca England appears, especially from an Asian perspective, only as a small spot on the world map. If the Scottish Government were to get their way and achieve independence from the United Kingdom the Brexit would prove to be a disastrous boomerang.
\end{abstract}

Schlüsselwörter Brexit - Continental alliance $\cdot$ Friedrich List and his vision of a German-English alliance · England's insular and trade supremacy · The British trade strategy and tactics

\section{Vorwort}

Nun sind also die Würfel gefallen! Das langwierige und mitunter unwürdige Tauziehen der englischen Regierungen um den Brexit hat ein Ende gefunden. Das Tischtuch ist aber nicht zerrissen, weil ein harter Brexit noch zu guter Letzt verhindert werden konnte. Jetzt beginnt eine neue Ära der britisch-europäischen Zusammenarbeit. Aus 
europäischer Sicht mag man den Brexit bedauern; aus englischer Sicht erscheint er konsequent.

Hierbei wird man daran erinnert, dass sich Friedrich List in seinem letzten Lebensjahr 1846 aus eigenem Antrieb auf den Weg gemacht hat, um der englischen Regierung und der Opposition eine deutsch-englische Allianz vorzuschlagen und wie enttäuscht er war, als er sich das Scheitern seiner Mission eingestehen musste. Deshalb drängt sich die Frage auf, wie List heute den Brexit kommentieren würde.

\section{Lists Bemühungen um eine deutsch-englische Allianz}

Nachdem List in der sog. zweiten Pariser Preisschrift von 1837 mit dem Titel „Le monde marche - Die Welt bewegt sich“, den Grundgedanken der Globalisierung entwickelt und diese Idee in seinen letzten Lebensjahren durch die „Politik der Zukunft“ visionär untermauert hatte, reifte in ihm im Frühjahr 1846 die Erkenntnis, dass sich aus den umwälzenden Auswirkungen der neuen Transport- und Kommunikationsmittel Dampfschifffahrt, Eisenbahn und Telegraphie zwangsläufig die Notwendigkeit einer deutsch-englischen Allianz aufdrängt. In Zukunft müsse es im Interesse Englands liegen, seine Industrie, den Handel und die Schifffahrt durch eine enge Kooperation mit Deutschland zu fördern, um gegenüber dem Machtanspruch von Frankreich, Russland und den USA ein Gegengewicht zu bilden. Deswegen sei eine enge wirtschaftliche, politische und kulturelle Zusammenarbeit zwischen England und dem politisch zunächst noch unvereinten Deutschland zwingend geboten.

Von dieser Idee geleitet, fasste er den Plan, nachdem er bereits 1824 zum ersten Mal englischen Boden betreten hatte, eine zweite Reise in die englische Hauptstadt zu unternehmen, um die englische Regierung mit dem amtierenden Premierminister Robert Peel und die parlamentarische Opposition unter Lord Palmerston für den Plan einer deutsch-englischen Allianz zu gewinnen (Wendler 2013, S. 289-300). Diese Mission kommentierte Gustav Kolb, der damalige Herausgeber der „Allgemeinen Zeitung“, Freund und Vertrauter von List, mit den Worten: „Ehe List nach England gegangen war, hatte ich einige sehr ernsthafte Unterredungen mit ihm gehabt, worin ich ihm vorstellte, wie er unmöglich in England seinen Zweck erreichen könne. Er beharrte dabei, diese sei die glorreichste Mission seines Lebens und der Zweck sei ein so großartiger, dass er sich schon belohnt fühle, ihn erstrebt zu haben." (Kolb 1849, S. 854)

List war also nicht von seinem Vorhaben abzubringen, obgleich ihm der gesunde Menschenverstand sagen musste, dass dieser Plan von vornherein zum Scheitern verurteilt war. Immerhin fand er in Anton Bachmaier, dem Mitinhaber des Handelshauses F. J. Bachmaier in Vilshofen, einen finanziellen Unterstützer dieser Reise. Am 16. März 1846 schrieb ihm Bachmaier: ,Ich wiederhole Ihnen, dass ich ganz Ihrem Dienste geweiht bin und keinen schöneren Lohn finde, als das Bewusstsein, durch meine Hilfe auch einen Stein zum großen Werk, das Sie mit edler Aufopferung und wahrem Patriotismus schon seit Jahren bauen, beitragen zu können." (Bachmaier 1846)

Anton Bachmaier startete sogar einen Aufruf zur Gründung eines Komitees der bedeutendsten Industriellen, Kaufleute und Großgrundbesitzer mit dem Ziel, Mit- 
tel einzuwerben, um Lists Reise nach England zu finanzieren. Dieser Aufruf war sogar recht erfolgreich, weil die nicht unbedeutende Summe von 6000 Gulden zusammenkam. Kolb berichtet, dass List diese Mittel nicht angetastet und bei einem Bankier hinterlegt hat. Erst nach seinem Tod hat Kolb die Summe dessen Witwe ausgehändigt (Bachmaier 1846, S. 799, 802).

Am 19. Juni 1846 traf List in London ein. Im Hotel Royal in der Bridgestreet, Blackfriars, quartierte er sich für zwei Monate ein. Das Hotel wurde vor 1845 als Luxushotel gebaut und existierte bis zum ersten Weltkrieg; es beherbergte vorwiegend Besucher aus dem europäischen Ausland (Wendler 1989, S. 206-221).

Unter der Mithilfe von einigen einflussreichen Persönlichkeiten, wie dem preuBischen Gesandten am englischen Hof, Freiherr v. Bunsen, dem einflussreichen Nationalökonomen und Politiker John Bowring, Prinz Albert, dem Gemahl der Königin Victoria, Fürst Karl v. Leiningen, dem Halbbruder der Königin und Baron v. Stockmar, einem engen Vertrauten der Monarchin, gelang es List, seine „Allianzdenkschrift" den maßgeblichen englischen Politikern zuzuleiten. Darin bekräftigt er seine Überzeugung, dass die Tage der englischen Wirtschaftssuprematie im Welthandel gezählt sind und die USA mit ihrer Wirtschaftskraft das Inselreich in absehbarer Zeit überflügeln werden, wenn die englische Regierung dieser Entwicklung tatenlos zusehe. Während die Produktivkräfte der USA in geometrischer Form wachsen, nähmen die englischen nur in arithmetischer Weise zu. Diesem Dilemma könne England durch zweckdienliches politisches Handeln und außerordentlichen Anstrengungen erfolgreich entgegenwirken. Für England gäbe es nur zwei Wege, die Suprematie zur See und im Welthandel gegen die rasch wachsende Macht der USA zu behaupten: England müsse entweder Mittel und Wege finden, um die nordamerikanische Union zu sprengen oder einen Plan entwickeln, wie es noch schneller an Reichtum und Macht wachsen könne als die Vereinigten Staaten. Die zweite Alternative halte er für die Wahrscheinlichere.

England werde durch die Entwicklung der USA angespornt, im gleichen Verhältnis mitzuziehen. Die hierzu erforderlichen Mittel werde es vor allem aus der Festigung und Ausdehnung seines Kolonialreiches ziehen. Je mehr seine Kolonien und Besitzungen in Asien, Afrika und Ozeanien an Bevölkerung, Zivilisation und Wohlstand zunähmen, desto größer werde die Einfuhr an Lebensmitteln und Rohstoffen, desto größer sein Absatz an Fertigfabrikaten, desto bedeutender seine Bevölkerung, sein Reichtum, seine Finanzkraft, seine Schifffahrt und folglich seine See- und Landmacht. Man könne damit rechnen, dass sich England bis China eine „Weltgasse“ bahnen und die „Häuser“ rechts und links davon seiner Herrschaft einverleiben werde. Man denke hier nur an Gibraltar, Malta, Zypern, Aden, Indien, Ceylon, Singapur und Hong Kong.

Kein Mensch könne voraussagen, wann dieses Ziel erreicht sei. „Aber das darf man keck sagen: das Menschenkind ist bereits geboren, das alles dies ausgeführt sehen wird.“ England werde überall in der Welt „Stapelplätze für seinen Handel“ anlegen. Außerdem werde es versuchen, diesen Ländern seine Kultur und seine Sprache der an Unterwürfigkeit und Arbeit gewöhnten Bevölkerung aufzuzwingen. Lediglich bei den Arabern rechne er hierbei mit größeren Schwierigkeiten.

List prophezeite aber gleichzeitig, dass das englische Weltreich nicht von Dauer sein werde. Er hielt es für sicher, dass „Australien, Neuseeland und die Kolonien an 
der Küste im südlichen und östlichen Afrika“ eine politische Bedeutung erlangen und nach dem Beispiel der Vereinigten Staaten ihre Unabhängigkeit erhalten werden.

Um seine Ziele zu erreichen, müsse England viel daran gelegen sein, eine Allianz mit Deutschland einzugehen; aber nicht mit Deutschland wie es gegenwärtig sei, sondern mit „Deutschland, wie es sein sollte und wie es mit Hilfe Englands werden könne;“" - ein nützlicher und potenter Alliierter könne Deutschland den Engländern aber nur dann sein, wenn seine nationale Wiedergeburt unter der Leitung einer eigenen Regierung vonstattengehe.

„Gewiss, England kennt die künftige Bedeutung Deutschlands nicht. Stolz auf seine gegenwärtige Übermacht, hat es sich noch nicht die Mühe gegeben, sich eine klare Vorstellung davon zu machen, welchem Schicksal diese Nation bei der Fortdauer ihrer gegenwärtigen Zersplitterung entgegengeht und welchen Einfluss diese bisher von ihm so gering geachtete Nation auf sein eigenes Geschick zu üben bestimmt ist."

Der deutsche Zollverein sei der vernünftige Vorläufer der politischen Einheit. „Handelseinigung und politische Einigung sind Zwillingsschwestern; die eine kann nicht zur Geburt kommen, ohne dass die andere folgt." England müsse aber dafür Verständnis zeigen, dass die deutsche Industrie, die eigentlich erst fünfzehn Jahre alt ist, gegenüber der fortgeschrittenen englischen Wirtschaft ebenso wenig bestehen könne, wie ein noch nicht erwachsener Knabe gegenüber einem starken Manne. Deutschland könne aber nur dann ein kräftiger und wirksamer Alliierter Englands werden, wenn es ein wohlorganisiertes, ein reiches und mächtiges Land ist. Dabei lehnte List einen Einheitsstaat nach französischem Vorbild ab und plädierte stattdessen für ein föderales System, weil er bei einem Einheitsstaat militärische Konflikte mit den Nachbarn befürchtete.

Es spreche alles dafür, die deutsch-englische Zusammenarbeit zu intensivieren und eine politisch-ökonomische Allianz anzustreben, die nur zum beiderseitigen Vorteil gereichen könne. England möge die Bemühungen Preußens unterstützen, die noch nicht dem Zollverein angehörenden deutschen Staaten mit Ausnahme von Österreich handelspolitisch zu integrieren. Ferner sollte es Deutschland beim Ausbau seiner produktiven Kräfte helfen und ihm das Zustandekommen der politischen Einheit in Frieden und Freiheit erleichtern. Als wirksamstes Mittel dieser Unterstützung rief List die englische Regierung dazu auf, ein temporären Schutzsystem zu tolerieren bis Deutschland ein ebenbürtiger Handelspartner geworden sei.

Dass diese Mission schon vom Ansatz her als Fehlschlag enden musste, versteht sich von selbst. List besaß weder ein politisches Mandat, noch konnte er auf die breite Unterstützung der öffentlichen Meinung in den deutschen Territorialstaaten zählen und zudem lag die politische Einheit Deutschlands aus englischer Sicht noch in weiter Ferne. Deswegen fielen auch die Stellungnahmen des englischen Premierministers Robert Peel und des Oppositionsführers Lord Palmerston ablehnend aus.

Peel ließ List wissen, dass er die Denkschrift aufmerksam gelesen habe. In Bezug auf die vorgeschlagene enge Allianz zwischen England und Deutschland stimme er mit ihm vollkommen überein, weil eine solche von beiderseitigem Vorteil wäre. Er halte aber die damit verknüpfte Zollpolitik für ganz und gar verfehlt. Wenn List die Beibehaltung hoher Einfuhrzölle für englische Produkte verlange, so widerspre- 
che dies der Allianzidee. Der englische Premier sprach sich stattdessen für einen raschen Abbau der Zollschranken und einen ungehinderten Warenaustausch aus. Außerdem äußerte er Zweifel an Lists Behauptung, dass er die Meinung der Mehrheit des deutschen Volkes vertrete. Er wünsche sich zwar auch eine enge deutsch-englische Kooperation, könne aber Lists falsche Ansichten den herrschenden politischen Autoritäten seines Landes nicht zur Annahme vorschlagen.

Dazu meine Gustav Kolb: „Peel nahm die Denkschrift kühl auf, indem er in einem höflichen Billet an List bemerkte: es mögen ihn gute Absichten geleitet haben, aber durch solche Prohibitiv- und Sperrmaßregeln würde das Glück der Völker nicht gefördert und England könne daher zu Lists Absichten nicht die Hand reichen.“

„Noch die meiste Aufmerksamkeit scheint er bei Lord Clarendon gefunden zu haben." Georges William Frederick Villiers, 4th Earl of Clarandon, wie sein voller Name lautet, war damals Präsident des Board of Trade, also englischer Handelsminister. Immerhin hatte ihn die Allianzdenkschrift so angesprochen, dass er List zu einer persönlichen Aussprache eingeladen hat.

In einer ausführlichen Stellungnahme brachte Oppositionsführer Lord Palmerston weitere Argumente zugunsten der englischen Freihandelsdoktrin vor. Wie Robert Peel bescheinigte auch Palmerston, dass Lists politische Ansichten wohl begründet, aber seine wirtschaftspolitischen Schlussfolgerungen falsch seien. Wenn List die Meinung vertrete, dass England mit Deutschland eine dauerhafte Allianz zum Wohle beider Nationen eingehen sollte, so sei dies unstrittig. Wenn er aber gleichzeitig die Forderung erhebe, dass Deutschland zur Erlangung seiner politischen Einheit vorläufig auf den Freihandel verzichten und stattdessen eine Schutzzollpolitik betreiben müsse, so sei dies ein Irrweg. Freihandel beginne wie die Nächstenliebe im eigenen Haus, und wenn List die Vorteile des Zollvereins für die wirtschaftliche Entwicklung Deutschlands zurecht lobe, so beruhe doch gerade dieser Erfolg auf den Prinzipien des Freihandels. Die besten Beispiele seien Bayern und Württemberg, die durch den Zollverein einen großen wirtschaftlichen Aufschwung erlangt haben. Schutzzölle seien für die Produktion wie für die Mehrung des Wohlstands einer Nation gleichermaßen von Nachteil. Somit könnten protektionistische Maßnahmen ein Land weder wohlhabend noch stark machen.

Insofern war diese Mission ein totaler Misserfolg. Resignierend stellte List fest, dass die englische Regierung weder die allgemeine Stimmung der Deutschen kenne, noch die langfristigen Auswirkungen ihrer ablehnenden Haltung für das eigene Land ,gehörig erwogen“ habe (List 1846, S. 263-296). Er kehrte, wie Gustav Kolb berichtet, ,entmutigt, fast möchte er sagen, gedemütigt nach Deutschland zurück“. Von diesem Tiefschlag hat sich List nicht mehr erholt und ein Vierteljahr später seinem Leben ein Ende gesetzt. Trotz seiner Kritik an dieser Mission kommentierte Kolb die Denkschrift mit den Worten: „Sie ist die ruhigste, folgerichtigste, geistvollste Darstellung, die je aus Lists Feder gekommen ist und die doch eben in jener Zeit geschrieben war, in der wir jene bedenklichen, geistigen Erscheinungen bei ihm beobachteten." (Kolb 1849, S. 854) 


\section{Die englische Insular- und Handelssuprematie}

Einen Aufsatz von 1843 leitete List mit den Worten ein: „Die Engländer sind eine große und achtbare Nation, die der Deutsche unter allen Nationen am meisten schätzt, mit welcher er am meisten sympathisiert und von der er selbst am besten verstanden, am gerechtesten und richtigsten beurteilt wird. Die Engländer als Individuen sind aufrichtig, rechtlich, wahrheits- und gerechtigkeitsliebend, großmütig, verständig, tüchtig in allem. Aber als Nation sind sie dies nur bis zu dem Punkt, wo ihr Handelsmonopol in Spiel kommt. Von da an sind sie, wie es alle ihre Vorgänger in Bezug auf die Handelsherrschaft waren und wie zweifelsohne alle ihre Nachfolger sein werden: ungerecht, hinterlistig, insolent (d. h. unverschämt und anmaßend), unersättlich in der Habsucht - ein Harpax unter den Nationen." (List 1843a, S. 250f.). Ein Harpax ist eine römische Waffe, die von Land aus wie eine Harpune abgefeuert wurde, um feindliche Schiffe zu entern.

Die Insellage von Großbritannien, die überragende Wirtschaftskraft und Handelsmacht, die das Land zur stärksten Wirtschaftsnation werden ließen, bezeichnete List als Insular- und Handelssuprematie. In dieser Hinsicht unterscheide sich England von allen anderen Nationen. In Bezug auf Ackerbau und Industrie, auf Flotte und Kolonien, auf inneren Reichtum und Bedeutung des Außenhandels sowie dem technischen Entwicklungsstand der Maschinen und Transportmittel stehe das Land weit über jeder anderen Nation. Die Fabriken der Handels- und Gewerbssuprematie hätten tausend Vorteile, z. B. geschickte und eingeübte Arbeiter in größter Zahl und zu billigsten Löhnen, die besten Techniker, die vollendetsten und wohlfeilsten Maschinen, die größten Vorteile beim Ein- und Verkauf, insbesondere die günstigsten Transportmittel zum Bezug der Rohstoffe und zur Versendung der Fertigfabrikate, großen Kredit bei den Banken zu niedrigen Zinsen, Erfahrungen, Werkzeuge, Gebäude, Anlagen und Geschäftsverbindungen, wie sie nur im Verlauf von Menschenaltern zu sammeln und herzustellen sind; dazu ein unermesslicher Binnenmarkt und ein ebenso unermesslicher Kolonialmarkt (List 1841, S. 315f., 349, 369).

In England habe sich die bürgerliche Freiheit früher entwickelt als in anderen Ländern. Die Interessen der Monarchie, der Aristokratie und der Demokratie haben sich dort früher als anderwärts verbunden und vereinigt, mit dem allgemeinen Ziel, die produktiven Kräfte der Nation im Innern und den Handel mit den Bewohnern in allen Himmelsrichtungen zu vermehren. Dort sehe man die Agrikultur-, die Manufakturund die Handelskräfte in höchster Vollkommenheit und schönstem Gleichgewicht. Dort habe man die praktische Anwendung der Douanengesetze durch die insulare Lage machtvoll unterstützt. Dort sehen wir, wie seit Jahrhunderten, abgesehen von einer kurzen Zwischenzeit, die Entwicklung und der Fortschritt der produktiven Kräfte stetig ihren Gang genommen haben. Dort beobachten wir, wie in der heimischen Industrie ein ewiger Friede herrscht, wie die gewaltige Überlegenheit der Seemacht die Grundlage bietet, den Außenhandel noch weiter auszubauen.

In Anbetracht des verfügbaren Kapitals bezeichnete List ,die englische Nation als die Bankhalterin aller Nationen des Erdballs“. Eine Nation aber, die stärker als alle anderen Nationen fabriziert, werde sich stets durch ihre Eigenliebe und ihren Nationalegoismus überhöhen und zum nationalen Eigendünkel neigen. Obwohl es die Vernunft gebiete, auf die ständig wachsende Eifersucht und Missgunst zu 
verzichten und obwohl diese Erkenntnis einem sage, dass der Krieg zwischen den Völkern ebenso töricht wie grausam ist und einem bewusst sei, dass der ewige Friede und die Handelsfreiheit alle Völker auf die höchste Stufe von Reichtum und Macht zu erheben vermögen, sei die Bereitschaft zu friedlicher Kooperation und internationaler Arbeitsteilung noch äußerst unterentwickelt. Das beste Mittel, um die internationalen Wirtschaftsbeziehungen zu verbessern und $\mathrm{zu}$ vertiefen, seien Handelskongresse, zu denen die kompetentesten Nationalökonomen ihrer Länder entsandt werden, um darüber zu beraten, welche gemeinsamen Interessen und Ziele vorrangig verfolgt werden sollten.

Damit wolle er keineswegs behaupten, dass es keine Suprematie geben dürfe und noch viel weniger, dass eine andere als die Suprematie Englands wünschenswert sei. In der Ordnung der Natur scheine es nun einmal zu liegen, dass die Gewächse der Politik genauso verschieden sind, wie die Gewächse der Natur, und sein Verlangen gehe nicht so weit, dass alle Bäume der Erde Eichen sein sollten. Er erhebe nur die Forderung, dass ,die anderen europäischen Nationen nicht von England und seinen Tochternationen derart überragt werden, dass sie daran gehindert werden, sich um ihre eigene Achse zu drehen und ihre eigene Bahn zu verfolgen - mit einem Wort, dass sie nicht zu bloßen Satelliten Englands herabgewürdigt werden. Gleichwohl lasse sich England durch seine Habsucht dazu verleiten, Nationen, die zur harmonischen Ausbildung ihrer Nationalkräfte berufen sind, in ihrem Werdegang aufzuhalten und zu stören, freilich nicht durch die Anwendung roher Gewalt, sondern was noch gefährlicher sei, durch die Benutzung einer falschen Doktrin sowie durch Überredung und diplomatische Künste. Lediglich in dieser Hinsicht sei er ein ernster Gegner von England“ (List 1837, S. 220-25).

„Man sieht klar, wie ein kollektives Kontinentalsystem im Werden ist. Und abermals sehen wir England alle Mittel, erlaubte wie unerlaubte, aufbieten, um seine alte Suprematie zu behaupten.“ (List 1839, S. 99). In einem Gedicht über ,John Bulls Rat an den deutschen Michel“ gießt List die egoistische „Handelslist der Briten“ in eine poetische Form und kritisiert andererseits den deutschen Michel wegen seiner Schlafmützigkeit.

\section{John Bulls Rat an den deutschen Michel}

Schlafe mein Kindchen, schlaf' ein! Fabriken, mein Herzchen, lass' sein! Und Besen und Lumpen und Stein Kauf' ich in Menge bei Dir ein.

Schlafe mein Kindchen, schlaf' viel! Und Zucker die Menge aus Brasil' Bringt Aberdeen Dir und mein Peel Geschmeide Deiner Amme gar viel.

Und wirst Du mein Kindchen nicht wach, Und fühlst Du Dich elend und schwach, 
Oh! so schreie nicht Wehe und Ach!

Sei freundlich und fröhlich und lach'!

Und wirst Du einst groß, oh so geh', Ich beschwöre Dich, niemals zur See!

Zur See ist's so grausig, herrjeh!

Da wird Dir so wind und so weh.

Verlasse doch niemals den Strand, Pflanz' Du dort mit fleißiger Hand Kartoffeln in Deinem Sand.

Nähre Dich ehrlich und redlich im Land!

Mit Aberdeen ist George Hamilton Gordon, 4th Earl of Aberdeen gemeint, der zu dieser Zeit englischer Außenminister war und mit Peel, der zur selben Zeit regierende englische Premierminister Robert Peel. In diesem Gedicht machte List den Briten unverhohlen den Vorwurf, dass sie ihre ökonomische und politische Suprematie zum Schaden der deutschen Territorialstaaten bzw. der Staaten des Zollvereins einsetzen, aber diese nichts dagegen unternehmen und somit ihre eigenen Interessen wegen der egoistischen Kleinstaaterei mit zu wenig Nachdruck verfolgen.

Dem berühmten Chemiker Justus Freiherr v. Liebig (1803-1875) ist Lists Wirken nicht unbekannt geblieben. Einem Freund schrieb er: „Niemand beurteilt die englischen Verhältnisse, die ich ziemlich gut durch meine Reisen dahin kenne, besser wie Herr List, aber welche Kluft zwischen seinen und den akzeptierten Ansichten unserer Staatsmänner.“ (Lenz und Wiskemann o.J., S. 623). Liebig dürfte sich hierbei auf Lists Zollvereinsblatt bezogen haben, das in zahlreichen Beiträgen über die politische und wirtschaftliche Situation auf der Insel berichtete.

\section{Die wirtschaftliche Vereinigung in Europa}

Gleichwohl dachte List schon damals an die Notwendigkeit einer Vereinigung von selbstständigen europäischen Staaten, um das anzustreben, was man das „Europäische Staatensystem“ oder das „Europäische Gleichgewicht“" zu nennen pflege. In der Einleitung eines dreiteiligen Beitrags zum Thema „Idées sur les réformes économiques, commerciales et financières, applicables à la France“, den er während seines mehrmonatigen Europaaufenthaltes im Jahre 1831 in der „Revue Encyclopédique“ veröffentlichte, plädierte er für „le modèle d'une bonne administration et préparer l'équlibre futur de l'Europe, fondé sur la sainte alliance des peuples." (List 1831, S. 60)

Bereits in seiner Denkschrift an die Bundesversammlung zur Abschaffung der Binnenzölle vom 20. April 1819 äußerte List die Überzeugung: Nur durch die allgemeine Handelsfreiheit könne Europa „,den höchsten Grad an Zivilisation erreichen“. „Mit neidischen Blicken sehe er über den Rhein, wo ein großes Volk vom Ärmelkanal bis zum Mittelmeer, vom Rhein bis zu den Pyrenäen, von der Grenze Hollands bis Italien auf freien Flüssen und offenen Landstraßen Handel treibe, ohne eine Zoll- 
grenze passieren zu müssen“, und an anderer Stelle vertrat er die Meinung: „Nur ein starkes und reiches Deutschland wird imstande sein, das Gleichgewicht auf dem Kontinent zu behaupten; ein armes und ausgesogenes wird jedem Anfall, ob er vom Osten oder Westen komme, unterliegen.“ (List 1842, S. 226)

In einer anderen Denkschrift, die List in seiner Mission als Delegierter des Handels- und Gewerbsvereins dem österreichischen Kaiser Franz I. am 2. März 1820 überreichte, schreibt er: „Die Theoretiker werden uns doch zugeben müssen, dass es dem deutschen Nationalwohlstand sehr förderlich wäre, wenn alle europäischen Staaten den deutschen Produkten offen stünden und, dass entsprechende Handelsverträge sehr wünschenswert wären.“

Nach seiner Verurteilung durch den Kriminalgerichtshof in Esslingen wegen der „Reutlinger Petition“ flüchtete List zunächst nach Straßburg. Dort begründete er eine Schriftenreihe, der er nach einer griechischen Sagengestalt den Namen „Themis“ gegeben hat. Sie gilt als Göttin der Gerechtigkeit, Sitte und Ordnung. Im Vorwort schreibt er u. a.: „Kein falscher Nationalstolz hält in unseren Tagen die Völker davon $\mathrm{ab}$, sich fremde Erfahrungen als Lehre dienen zu lassen und auswärts erprobte Institutionen im eigenen Lande zu errichten“, denn so fügte er hinzu: „Meine Augen sind auf Europa gerichtet!“

Das Haupthindernis für eine engere ,Vereinigung des europäischen Kontinents“ sah List in der desolaten Lage des wirtschaftlich und politisch zersplitterten Deutschlands. „Anstatt Vermittler zwischen dem Osten und Westen des europäischen Kontinents in allen Fragen der Gebietseinteilung, des Verfassungsprinzips, der Nationalselbstständigkeit und Macht zu sein, wozu dasselbe durch seine geographische Lage, durch eine Föderativverfassung, die alle Furcht vor Eroberung bei benachbarten Nationen ausschließt, durch seine religiöse Toleranz und seine kosmopolitischen Tendenzen, endlich durch seine Kultur- und Machtelemente berufen ist, bildet dieser Mittelpunkt den Zankapfel, um den sich der Osten und Westen streiten, weil man beiderseits, diese durch Mangel an Nationaleinheit geschwächte, stets ungewiss hin und her schwankende Mittelmacht auf seine Seite zu ziehen hofft. Würde dagegen Deutschland mit den dazu gehörigen Seegestaden, mit Holland, Belgien und der Schweiz sich als kräftige kommerzielle und politische Einheit konstituieren und würde dieser mächtige Nationalkörper mit den bestehenden monarchischen, dynastischen und aristokratischen Interessen die Institutionen des Repräsentativsystems miteinander verschmelzen, soweit sie miteinander in Einklang zu bringen sind, so könnte Deutschland dem europäischen Kontinent den Frieden für lange Zeit verbürgen und zugleich den Mittelpunkt einer dauerhaften Kontinentalallianz bilden.“ (List 1837, S. 40)

In einer derart geeinten und liberalisierten europäischen Wirtschaftsunion werde Deutschland die große kommerzielle Verbindungsstraße zwischen dem östlichen und westlichen und zwischen dem nördlichen und südlichen Europa bilden und unter dem Schirm des äußeren und inneren Friedens seinen Wohlstand aufbauen, wenn die Zollgrenzen und der allgemeine deutsche Handels- und Gewerbsverein und der Name seines geistigen Urhebers, also Lists Name, schon längst in Vergessenheit geraten sind.

Durch die neuen Kommunikationsmittel könne sich der Absatzmarkt jedes Produzenten und jedes Händlers, den Thünen'schen Kreisen entsprechend, ausweiten und 
sich die Produktion wie der Konsum der proportionalen Ausdehnung des Marktes entsprechend erhöhen, sodass sich der internationale Handel schließlich über den ganzen Erdball ausbreiten werde. Hier ist also die Globalisierung vom Ansatz her vorausgedacht.

Die Eisenbahn werde auf dem europäischen Kontinent wie ein eisernes Band wirken und den endgültigen Zusammenschluss von Wirtschaftsräumen und Kulturzonen, wie etwa den drei Sprachgebieten der Schweiz, herbeiführen. Der europäische Kontinent, der von Cadix und Lissabon bis St. Petersburg und Moskau und von Neapel bis Le Havre über Paris, wo der Eisenbahnknotenpunkt, das Zentrum dieser beiden riesenhaften Linien liegen werde, und von Paris quer durch Deutschland, Österreich-Ungarn bis zum Schwarzen Meer, werde seinen Handel in einem ungeheuren Umfang erweitern, ohne von einer maritimen Großmacht abhängig zu sein. Diese Formulierung spielt natürlich auf England an.

\section{Die politische Vereinigung in Europa}

Schließlich werde der Zeitpunkt kommen, an dem das in zahlreiche kleine Bruchstücke zersplitterte Europa auch eine politische Vereinigung anstreben müsse. Diese Notwendigkeit ergebe sich nicht zuletzt aus zwei Gründen:

Russland zählte für List zwar nur bedingt zur ersten Klasse der Industrienationen, weil seine Stellung als Weltmacht noch nicht gesichert sei; dennoch befürchtete er, dass sich das Land vor allem auf seine militärische Macht stützen werde. Es bestehe die Gefahr, dass dieser Koloss damit fortfahre sein Riesenreich nach Westen auszudehnen, soweit dies die westliche Zivilisation zulasse. Daraus ergebe sich die Gefahr, dass Russland versuchen werde, die Staaten Mittel- und Westeuropas unter seine Kontrolle zu bringen. Diese Unterjochung werde aber nicht eintreten, wenn sich diese Länder wirtschaftlich und politisch zusammenschließen würden.

Eine andere Bedrohung für Europa sah List in einem möglichen Bevölkerungsdruck von Afrika. Wenn man es nicht schaffe, die Bevölkerungsentwicklung dieses Kontinents durch eine entsprechende wirtschaftliche Entwicklung aufzufangen, dann könnte das für Europa irgendwann noch gefährlich werden. So schreibt er in einem Artikel des Staats-Lexikons von 1834: Wenn es nicht gelinge die Hauptgründe für die Rückständigkeit des afrikanischen Kontinents zu beseitigen, könnte eines Tages die Gibraltar gegenüberliegende spanische Exklave ,,Tanger mit dem Fort Ceuta bei einer Invasion von Marokko aus (für Europa) von Wichtigkeit werden." An Lampedusa, Libyen, Syrien und die Balkanroute hatte er freilich damals noch nicht gedacht.

Wahrscheinlich schwebte List allerdings nur eine politische Integration der Staaten vor, die wir heute mit dem Begriff „Kerneuropa“ bezeichnen: Deutschland, Belgien, Holland, Frankreich, die Schweiz und Dänemark. An die Ost- und Süderweiterung hatte er ganz bestimmt nicht gedacht, zumal etwa das Osmanische Reich noch bis an die Grenze der Habsburger Monarchie reichte und z. B. auch Italien noch nicht vereinigt war.

Hinsichtlich der Ost- und Süderweiterung der Europäischen Union ist darauf hinzuweisen, dass List für eine wirtschaftliche und politische Vereinigung zwei Vor- 
aussetzungen verlangte. Zum einen müsse sie freiwillig erfolgen und zum zweiten sollten sich nur solche Staaten zusammenschließen, die sich in etwa auf derselben Entwicklungsstufe befinden. Für Griechenland, Bulgarien, Rumänien und andere kritischen EU-Mitglieder ist die erste Voraussetzung zweifellos erfüllt. Ob aber auch die zweite Voraussetzung im Sinne von List für den Beitritt gegeben war, darf bezweifelt werden.

Aufgrund seiner hohen Zivilisation und seiner Ressourcen sollte sich nach Lists Meinung Frankreich an die Spitze der europäischen Einigungsbestrebungen stellen, weil es in politischer, rechtlicher, wirtschaftlicher und kultureller Hinsicht das fortschrittlichste Land auf dem europäischen Kontinent sei. Als Zentrum des europäischen Handels werde die französische Hauptstadt zum geographischen Schnittpunkt von Orient und Okzident sowie von Nord- und Südeuropa.

Dass für einen solchen Brückenschlag die belgische Hauptstadt ein wichtiger Brückenpfeiler wäre, deutete List schon 1831 in einem Brief an den amerikanischen Außenminister Van Buren an, in dem er die amerikanische Regierung um eine diplomatische Funktion in Belgien ersuchte und darauf aufmerksam machte, dass Brüssel ein Zentralpunkt zwischen Deutschland und Frankreich sei.

\section{Der Brexit und die Kontinentalallianz}

Nach den erfolglosen Bemühungen um eine deutsch-englische Allianz musste List zur Einsicht gelangen, dass man bei der europäischen Integration nicht auf England zählen kann. Schon vorher hatte er dies in entsprechenden Formulierungen angedeutet und zum Ausdruck gebracht. Einige Belege werden hier beispielhaft zitiert.

„Die Liebe zu Britannia ist wie die Liebe zu Jupiter: Wer sie umarmen will, wird von ihrem Feuer verzehrt wie Semele.“ (List 1844, S. 255). In der griechischen und römischen Mythologie ist Semele die Tochter der Göttin der Eintracht, Harmonia. Der Göttervater Jupiter erschien Semele als gewöhnlicher Sterblicher und verliebte sich in sie. Seine Gattin Hera wurde deshalb eifersüchtig, als sie von dieser Liebschaft erfuhr und säte Zwietracht, weshalb Semele Zweifel an ihrer Liebe kamen. Um diese auszuräumen, wünschte sie sich, dass sich Jupiter in seinem göttlichen Glanz zeigen möge. Als sich dieser Wunsch erfüllte, wurde Semele, wie ein Körper, welcher der Sonne zu nahekommt, vom Blitz getroffen und von der Hitze versengt.

Im neunten Brief seiner „Outlines of American Political Economy“ meint List: Je mehr die englische Politik zum gegenwärtigen Zeitpunkt sich selbst zu widersprechen scheint und ihre Entscheidungen in unheilvolles mystisches Dunkel hüllt, desto schwieriger sei es, deren Haltung zu verstehen. Frage man nach der Absicht der englischen Regierung, so zeige sich, dass diese darin besteht, die eigene Wirtschaftsmacht so zu stärken, dass kein anderes Land mehr mit England konkurrieren kann. Um dieses Ziel zu erreichen, unterstütze die Regierung daheim liberale Grundsätze, während sie in Asien den Eroberer spielt und die dortigen despotischen Regierungen stützt und sich auf den westindischen Inseln und in Kanada mit einer väterlichen Regierung begnügt. Stets passe sie ihr Verhalten den Umständen an, indem sie zu einer Zeit und an einem Ort liberale Grundsätze anwendet, dann wieder Macht und Geld einsetzt, um entweder die Freiheit zu fördern oder zu unterdrücken, wie es 
ihren Zielen dienlich ist. List begnügte sich mit dieser Andeutung, weil er nicht die Absicht habe, die europäische Politik abzuhandeln. Er wolle lediglich darlegen, dass die englische Politik immer das Ziel verfolge, den europäischen Kontinentalmächten entgegenzuarbeiten (List 1827, S. 120-22).

Andererseits gab er zu bedenken: „Die Kraft Deutschlands ist die Kraft Englands“ (List 1845, S. 263). Jedoch: „Man zeigt Deutschland die Gefahren, von welchen es rechts und links bedroht ist, man lässt Deutschland fühlen, wie wünschenswert, wie notwendig ihm die Freundschaft und Hilfe Englands sei und als Handgeld auf einen solchen Dienst verlangt man, Deutschland solle sein Streben nach Handelsund Gewerbsunabhängigkeit der Handelssuprematie zum Opfer schlachten." (List 1843a, S. 252)

Betrachte man die unermesslichen Interessen, welche die Kontinentalnationen besitzen, so müsse man erkennen, dass nun ein ,,veredeltes Kontinentalsystem“ (List 1840, S. 380) nottut. Eine solche „Kontinentalallianz“ könne nur Erfolg haben, wenn Frankreich nicht die Fehler Napoleons und - so fügen wir hinzu - Deutschland nicht die Fehler in der wilhelminischen Ära und im Nationalsozialismus begeht. „Ein wirksames Kontinentalsystem kann nur aus freier Vereinigung der Kontinentalmächte hervorgehen und nur Erfolg haben, wenn es die Gleichstellung der daraus erwachsenden Vorteile bezweckt und bewirkt." Man sehe klar, wie ein kollektives Kontinentalsystem im Werden ist; abermals werde dann England alle Mittel aufbieten - erlaubte wie unerlaubte - um seine alte Suprematie zu behaupten (List 1839, S. 99).

Allerdings werde es die nach Übermacht allzu durstigen Briten hart ankommen, wenn sie dann feststellen müssen, dass die Kontinentalallianz stärker an Wirtschaftskraft und politischem Einfluss gewinnt, als sie selbst. Dieselben Ursachen, die Großbritannien auf sein damaliges hohes Niveau gehoben haben, werden wahrscheinlich im Laufe des nächsten Jahrhunderts die USA und China auf eine Stufe heben, die England weit überragen wird. So werde in einer nicht allzu fernen Zukunft die Notwendigkeit, welche den Franzosen und den Deutschen die Stiftung einer Kontinentalallianz gebiete, es den Briten auch irgendwann nahelegen, sich (wieder) unter den Schutz, die Sicherheit und Geltung der europäischen Kontinentalnationen zu stellen. Es wäre also gut - so empfahl List - wenn sich England bei Zeiten die Freundschaft der europäischen Kontinentalmächte sichern und an den Gedanken gewöhnen würde, „,der Erste unter Gleichen zu sein!“ (List 1841, S. 415-17)

Was würde nun List in Anbetracht des Austritts von Großbritannien aus der EU und dem Abkommen zum Brexit den Deutschen raten? Er würde ihnen vielleicht sagen: „Sagen wir den Engländern: ihr braucht einen tüchtigen Alliierten auf dem Kontinent. Wir sympathisieren mit Euch in jeder Beziehung und in stärkerem Maße als mit jeder anderen, nicht der EU angehörenden Nation. Aber wir möchten gerne aufrichtige und tüchtige Alliierte sein. Deshalb müsst ihr aufhören, gegen unseren Aufschwung in Gewerbe, Handel und Schifffahrt zu intrigieren.“ (List 1843b, S. 254)

Funding Open Access funding enabled and organized by Projekt DEAL.

Open Access Dieser Artikel wird unter der Creative Commons Namensnennung 4.0 International Lizenz veröffentlicht, welche die Nutzung, Vervielfältigung, Bearbeitung, Verbreitung und Wiedergabe in jeglichem Medium und Format erlaubt, sofern Sie den/die ursprünglichen Autor(en) und die Quelle ord- 
nungsgemäß nennen, einen Link zur Creative Commons Lizenz beifügen und angeben, ob Änderungen vorgenommen wurden.

Die in diesem Artikel enthaltenen Bilder und sonstiges Drittmaterial unterliegen ebenfalls der genannten Creative Commons Lizenz, sofern sich aus der Abbildungslegende nichts anderes ergibt. Sofern das betreffende Material nicht unter der genannten Creative Commons Lizenz steht und die betreffende Handlung nicht nach gesetzlichen Vorschriften erlaubt ist, ist für die oben aufgeführten Weiterverwendungen des Materials die Einwilligung des jeweiligen Rechteinhabers einzuholen.

Weitere Details zur Lizenz entnehmen Sie bitte der Lizenzinformation auf http://creativecommons.org/ licenses/by/4.0/deed.de.

\section{Literatur}

Bachmaier, A. (1846). Brief an F. List. In W. VIII.

Kolb, G. (1849). Brief an L. Häusser. In W. VIII.

Lenz, F. und E. Wiskemann (o. J.). Kommentar zu W. VII.

List, F. (1827). Outlines of American Political Economy. Wiesbaden: Dr. Böttiger Verlags-GmbH.

List, F. (1831). Idées sur les réformes économiques, commerciales et financières, applicables à la France. In $I n W . V$.

List, F. (1837). Das natürliche System der politischen Ökonomie. In W. IV. Berlin: Reimar Hobbing.

List, F. (1839). Ankündigung und Plan einer Staatsbibliothek. In W. IX.

List, F. (1840). Wesen und Wert einer nationalen Gewerbsproduktivkraft. In W. $V$.

List, F. (1841). Das nationale System der politischen Ökonomie. In W. VI. Berlin: Reimar Hobbing.

List, F. (1842). Die deutsche Industrie. In $W . V$.

List, F. (1843a). Die englische Allianz und die deutsche Industrie. In W. VII.

List, F. (1843b). Die politische Nationaleinheit der Deutschen. In W. VII.

List, F. (1844). Die englische Handelsfrage vom deutschen Standpunkt aus betrachtet. In W. VII.

List, F. (1845). Die wahren Grundbedingungen einer deutsch-englischen Allianz. In W. VII.

List, F. (1846). Die wahren Grundbedingungen einer deutsch-englischen Allianz. In W. VII.

Wendler, E. (1989). Friedrich List-Politische Wirkungsgeschichte des Vordenkers der europäischen Integration. München: R. Oldenburg.

Wendler, E. (2013). Friedrich List (1789-1846) - Ein Ökonom mit Weitblick und sozialer Verantwortung. Wiesbaden: Springer Gabler. 